Project 1010098

\title{
Genes for Uranium Bioremediation in the Anaerobic Sulfate-Reducing Bacteria
}

\author{
Wall, Judy D. \\ University of Missouri
}

\begin{abstract}
RESULTS TO DATE: Cytochrome c3 interaction with U(IV):
Surprising results were obtained following an attempt to induce or derepress the machinery for $\mathrm{U}(\mathrm{VI})$ reduction by growing Desulfovibrio desulfuricans $\mathrm{G} 20$ in the presence of $1 \mathrm{mM}$ uranyl acetate. G20 cells grown on lactate-sulfate medium amended with $\mathrm{U}(\mathrm{VI})$ reduced uranium at a slower rate than cells grown in the absence of this metal. When periplasmic extracts of these cells were prepared, Western analysis of the proteins revealed that the cytochrome $\mathrm{c} 3$ was absent. This observation has been further investigated.
\end{abstract}

Molecular analyses of G20 cultures grown with uranium showed that the cytochrome was synthesized and was likely to be matured appropriately. After elimination of other alternatives, it was observed that cytochrome c3 released from the periplasm (or from whole cells) apparently bound tightly to the uraninite mineral produced through the reduction of $\mathrm{U}(\mathrm{VI})$. To test this hypothesis, purified cytochrome was added to chemically reduced uranium. Western analyses of the soluble and precipitated material containing uraninite showed that detectable cytochrome c3 was found only in the precipitated material. We interpret these results to mean that cytochrome $\mathrm{c} 3$ has a high affinity for the $\mathrm{U}(\mathrm{IV})$ precipitate. This may be a nonspecific hydrophobic interaction with the uncharged UO2 or possibly a specific interaction. Preliminary results suggest a non-specific interaction. Additional experimentation is needed to examine the effects of growth with uranium on subsequent reduction ability.

Nitrite reductase:

The hexaheme cytochrome $c$ of Shewanella putrefaciens been shown by mutational analysis to be responsible for uranium reduction by that bacterium (Wade and DiChristina, FEMS Microbiol. Lett. 184:143-148, 2000). The primary metabolic role of this protein is nitrite reduction in Shewanella, allowing that bacterium to grow with nitrate respiration. It apparently also allows growth with $\mathrm{U}(\mathrm{VI})$ as terminal electron acceptor. In the Desulfovibrio vulgaris Hildenborough genome sequence [made available to us by John Heidelberg of TIGR], two ORFs have been identified as 65 and $68 \%$ identical to NfrH and NfrA, small and catalytic subunits of nitrite reductase from D. desulfuricans ATCC 27774 (EMBL accession AJ316232.1). D. vulgaris appears to have two polypeptides, 159 and 524 residues respectively, which contain four and five hemes, respectively. We have assumed that $D$. desulfuricans has similar genes and have shown that strain $\mathrm{G} 20$ has nitrite reductase activity in whole cells.

To explore a possible involvement of nitrite reductase in the reduction of $\mathrm{U}(\mathrm{VI})$ in $\mathrm{D}$. desulfuricans, experiments were carried out to determine whether nitrite would compete for electrons available for $\mathrm{U}(\mathrm{VI})$ reduction. Sodium nitrite at $1 \mathrm{mM}$ did not detectably inhibit $\mathrm{U}(\mathrm{VI})$ reduction by wild-type $\mathrm{G} 20$ when the source of reductant was $10 \mathrm{mM}$ lactate, $10 \mathrm{mM}$ pyruvate, or one atmosphere of hydrogen gas. In the mutant lacking cytochrome c3, there was no inhibition with organic acid electron donors but the residual hydrogen supported $\mathrm{U}(\mathrm{VI})$ reduction was eliminated. It has been shown that nitrite irreversibly inhibits [NiFe] hydrogenase of Azospirillum brasilense (Tibelius and Knowles, J. Bacteriol. 160:103-106, 1984). Perhaps the remaining $\mathrm{U}(\mathrm{VI})$ reduction in the cytochrome c3 mutant is dependent on an orthologous protein. 
A complementary experiment explored whether nitrite reduction was inhibited by $\mathrm{U}(\mathrm{VI})$. There was no inhibition of nitrite reduction by $\mathrm{U}(\mathrm{VI})$ regardless of electron donor or presence or absence of cytochrome c3. Interestingly, during the course of these experiments, it was observed that the mutant lacking cytochrome $\mathrm{c} 3$ reduced nitrite at a faster rate with organic electron donors than did the wild type strain; CycA strain with lactate $0.64+0.04$ versus $\mathrm{G} 20,0.52+0.13 \mathrm{um} \mathrm{NO2-} \mathrm{reduced} / \mathrm{mg}$ cell protein $\mathrm{xh}$ and CycA strain with pyruvate $2.0+0.6$ versus G20, $1.0+0.36$ um NO2- reduced/mg cell protein $x \mathrm{~h}$. Our observations of the different activities in the mutant versus the wild type strain suggest that, in the mutant, the nitrite reductase may be increased in concentration or that electrons previously used through the missing tetraheme cytochrome c3 are now shunted through nitrite reductase.

A different result was obtained with hydrogen as the electron donor. While nitrite reduction by hydrogen was poor in the wild type cells $(0.15+0.06 \mathrm{um} \mathrm{NO2-} \mathrm{reduced/mg} \mathrm{cell} \mathrm{protein} \times h)$, reduction was not detected above the rate obtained without an electron donor in the cytochrome c3 mutant. Hydrogen supported reduction of nitrite by G20 would appear to be dependent on cytochrome c3 since this activity was eliminated in the mutant. Results of Liu and Peck with purified components from D. desulfuricans ATCC 27774 (J. Biol. Chem. 256:13159-13164, 1981) suggest the electron chain may be more complex. They were unable to demonstrate nitrite reduction from hydrogen in a system containing hydrogenase, cytochrome c3, and nitrite reductase. They interpreted this observation to mean that cytochrome c3 was unable to donate electrons to nitrite reductase. Thus it is likely that additional carriers are involved in the path to nitrite. A mutant of the nitrite reductase is being sought to confirm the role of this c-type cytochrome.

Regulatory systems of Desulfovibrio:

The availability of the genome sequence of $D$. vulgaris Hildenborough has offered the opportunity to analyze the regulatory features of this bacterium. We are employing a computational strategy to predict potential regulatory systems involved in metabolic regulons with the goal of applying these tools to genes involved in the reduction of $\mathrm{U}(\mathrm{VI})$ to $\mathrm{U}(\mathrm{IV})$. The strategy involves extracting the intergenic regions (igr) of the genome, grouping them by analogy to experimentally determined regulons from other bacteria, aligning the members of a group and examining them for conserved regions representing putative regulatory motifs. A small program was written to extract the igr sequences from the genome for comparison and libraries from the total chromosome have been constructed. Motifs that fall within statistical criteria will next be compared to known regulatory motifs of $\mathrm{E}$. coli to determine whether the motifs represent conserved or unique regulatory systems. Finally the results will be compared to microarray analyses of energy metabolism genes in the presence of uranium to predict which of these putative regulons are most likely to affect uranium reduction.

Currently orthologs for all amino acid metabolic pathways and central carbon metabolism have been identified. Potential regulons for these pathways have been identified by virtue of the existence of such regulons in other bacteria. The igr?s of these regulons will now be aligned and analyzed. We anticipate obtaining evidence for the ribosomal binding sequence and the ?sigma-70? promoter sequences as well as regulon-specific regulatory sequences. Each candidate sequence will need experimental confirmation.

DELIVERABLES: Reviewed publication:

Payne, Rayford B., Darren M. Gentry, Barbara J. Rapp-Giles, Laurence Casalot, and Judy D. Wall. Uranium reduction by Desulfovibrio desulfuricans strain G20 and a cytochrome c3 mutant. Appl. Environ. Microbiol. 68:3129-3132.

Abstracts:

Payne, Rayford B., and Judy D. Wall. 2002. Effect of Uranium on the cytochrome c3 of Desulfovibrio desulfuricans. Abstr. Amer. Soc. Microbiol. 102nd Gen. Meet., Salt Lake City, Utah, p. 448. 
Presentations:

Wall, Judy D., and Christopher L. Hemme. 2001. Metabolic insights of Desulfovibrio vulgaris derived from genomic information. 9th Internatl. Conf. Microbial Genomes, Gatlinberg, TN.

Posters: Hemme, Christopher L., and Judy D. Wall. 2002. Predicting the Genetic Regulation of Uranium Reduction in Desulfovibrio vulgaris. 2nd Annual TIGR-ASM Conference on Microbial Genomes, Los Vegas, NV.

Wall, Judy D., Rayford B. Payne, Suzanne Miller, Barbara Rapp-Giles. 2002. Genes for uranium bioremediation in the anaerobic sulfate-reducing bacteria. DOE-NABIR Grantee/Contractor Workshop, Warrenton, VA.

Miller, Suzanne M., Joseph A. Ringbauer, Jr., Barbara J. Rapp-Giles, and Judy D. Wall. 2002. Organization of respiratory genes in Desulfovibrio. Molecular Biology Week 2002. Univ. MissouriColumbia, Columbia, MO. 